\section{Commentary: What will make surgeons do more atrial fibrillation ablations?}

\section{Leonid Sternik, MD}

I wish to thank authors of this important multicentral study dealing with barriers to atrial fibrillation (AF) ablation during mitral surgery. ${ }^{1}$ The authors mention that nearly $40 \%$ of patients with AF undergoing mitral valve surgery do not receive concomitant ablation despite societal guidelines. Badhwar and colleagues ${ }^{2}$ reported in 2017 that surgical ablation during mitral surgery was performed in only $68.4 \%$ of patients with $\mathrm{AF}$ in the United States. Nine years earlier, Gammie and colleagues $^{3}$ published that a surgical AF ablation was done just in $52 \%$ (6415 of 12,235) of mitral valve surgeries in patients with AF. Although we have seen an increase in the rate of surgical concomitant $\mathrm{AF}$ ablation over the last decade, still too many patients with AF do not undergo an ablation during mitral surgery.

Mehaffey and colleagues ${ }^{1}$ reported that stratifying by mitral valve surgical volume, a majority of high-volume ( $\geq 50$ cases/year) and medium-volume (10-50 cases/year) surgeons reported no barriers in performing $\mathrm{AF}$ ablation. A significantly greater proportion of low-volume $(<10$ cases/year) mitral valve surgeons reported risk (eg, patients too high risk, additional crossclamp time, worsens arrhythmias) as a barrier to a concomitant ablation $(P=.023)$. Surgeons with no barriers report that they perform a concomitant Cox maze IV in $78 \%$ of open atrial operations compared with $27 \%$ for surgeons who report barriers. The authors of the commented paper conclude that knowledge of recommendations and practice patterns vary widely across surgeons. They provide specific interventions to

\footnotetext{
From the Department of Cardiac Surgery, Sheba Medical Center, Tel-Hashomer, Israel.

Disclosures: The author reported no conflicts of interest.

The Journal policy requires editors and reviewers to disclose conflicts of interest and to decline handling or reviewing manuscripts for which they may have a conflict of interest. The editors and reviewers of this article have no conflicts of interest.

Received for publication April 1, 2021; revisions received April 1, 2021; accepted for publication April 5, 2021; available ahead of print April 20, 2021.

Address for reprints: Leonid Sternik, MD, Department of Cardiac Surgery, Sheba Medical Center, Tel-Hashomer, Israel 52621 (E-mail: Leonid.sternik@sheba. health.gov.il).

J Thorac Cardiovasc Surg 2023;165:659-60

0022-5223/\$36.00

Copyright (c) 2021 by The American Association for Thoracic Surgery

https://doi.org/10.1016/j.jtcvs.2021.04.012
}

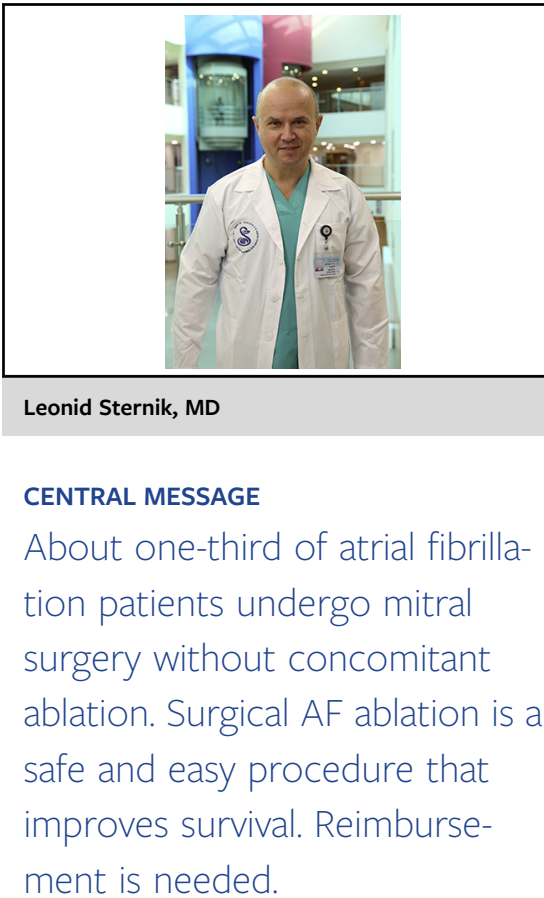

overcome these barriers, including mentorship, systematic protocols, peer-to-peer education, and additional research. ${ }^{1}$ I agree that high-volume surgeons usually do a better job. In contrast, an AF ablation is a relatively not complex surgery that can be performed easily almost by any surgeon. We have seen a lot of courses and proctoring dealing with $\mathrm{AF}$ surgical ablation in the last decade. I feel that we have a plenty of hard data proving the advantages of concomitant surgical AF ablation. The 2017 Society of Thoracic Surgeons guidelines assigned Class I, Level of evidence A for surgical AF ablation at the time of mitral surgery. Guidelines said that surgical ablation for AF can be performed without an additional risk of operative mortality or major morbidity. ${ }^{4}$ In a recent meta-analysis of randomized controlled trials, McClure and colleagues ${ }^{5}$ found that concomitant surgical ablation of AF had no significant effect on mortality. Suwalski and colleagues ${ }^{6}$ showed the survival benefit of surgical concomitant $\mathrm{AF}$ ablation on thousands of patients with long-term follow-up and strong statistical analysis. This survival benefit was maintained in all groups of patents. ${ }^{6}$ Petersen and colleagues ${ }^{7}$ reported that $\mathrm{AF}$ ablation is as effective in elderly patients as in younger patients in mitral surgery. No ablation-related complications were found. ${ }^{7}$ I agree with the authors' conclusion that education and proctoring are very important. However, we already have enough hard data proving that surgical 
concomitant $\mathrm{AF}$ ablation is a great procedure. I feel that what we need now is a reimbursement of this procedure.

\section{References}

1. Mehaffey JH, Charles EJ, Berens M, Clark MJ, Bond C, Fonner CE, et al. Barriers to atrial fibrillation ablation during mitral valve surgery. J Thorac Cardiovasc Surg. 2023;165:650-8.e1.

2. Badhwar V, Rankin JS, Ad N, Grau-Sepulveda M, Damiano RJ, Gillinov AM, et al. Surgical ablation of atrial fibrillation in the United States: trends and propensity matched outcomes. Ann Thorac Surg. 2017;104:493-500.

3. Gammie JS, Haddad M, Milford-Beland S, Welke KF, Ferguson TB Jr, O’Brien SM, et al. Atrial fibrillation correction surgery: lessons from the Society of Thoracic Surgeons National Cardiac database. Ann Thorac Surg. 2008;85:909-14.
4. Badhwar V, Rankin JS, Damiano RJ Jr, Gillinov AM, Bakaeen FG, Edgerton JR, et al. The Society of Thoracic Surgeons 2017 clinical practice guidelines for the surgical treatment of atrial fibrillation. Ann Thorac Surg. 2017;103:329-41.

5. McClure GR, Belley-Cote EP, Jaffer IH, Dvirnik N, An KR, Fortin G, et al. Surgical ablation of atrial fibrillation: a systematic review and meta-analysis of randomized controlled trials. Europace. 2018;20:1442-50.

6. Suwalski P, Kowalewski M, Jasinski M, Staromlynski J, Zembala M, Widenka K et al. Survival after surgical ablation for atrial fibrillation in mitral valve surgery. Analysis from KROK (Polish Nationwide Heart Surgery Registry). J Thorac Cardiovasc Surg. 2019;157:1007-18.

7. Petersen J, Vettorazzi E, Alassar Y, Meyer WS. Should concomitant surgical ablation for atrial fibrillation be performed in elderly patients? J Thorac Cardiovasc Surg. 2021;161:1816-23.e1.
See Article page 650.

\section{Commentary: Navigating a MAZE of implementation science for surgical atrial fibrillation ablation}

\author{
Alexander Iribarne, MD, MS,,${ }^{a, b}$ and \\ Kathleen Fenton, MD, MS
}

Contemporary trials in cardiovascular medicine focus on studying new treatments or techniques for existing diseases that impact public health. There is often a clear progression of knowledge from bench research to phase I clinical trials (translation to humans; T1), then phase II and III trials (translation to patients; T2), and finally phase IV trials (translation to clinical practice; T3). However, significant gaps exist in our understanding of how research findings are disseminated and implemented in common practice

\footnotetext{
From the ${ }^{\text {a Section }}$ of Cardiac Surgery, Dartmouth-Hitchcock Medical Center, Heart \& Vascular Center, Lebanon, NH; ${ }^{\mathrm{b}}$ The Dartmouth Institute for Health Policy and Clinical Practice, Dartmouth College, Lebanon, $\mathrm{NH}$; and ${ }^{\mathrm{c}}$ National Heart, Lung, and Blood Institute, National Institutes of Health, Bethesda, Md.

Disclosures: Dr Fenton is an employee of the National Heart, Lung, and Blood Institute (NHLBI), National Institutes of Health (NIH). The views expressed in this article are those of the authors and do not necessarily represent the views of the NHLBI, the NIH, or the US Department of Health and Human Services. Dr Iribarne reported no conflicts of interest.

The Journal policy requires editors and reviewers to disclose conflicts of interest and to decline handling or reviewing manuscripts for which they may have a conflict of interest. The editors and reviewers of this article have no conflicts of interest.

Received for publication April 11, 2021; revisions received April 11, 2021; accepted for publication April 12, 2021; available ahead of print April 18, 2021.

Address for reprints: Alexander Iribarne, MD, MS, Division of Cardiac Surgery, Dartmouth-Hitchcock Medical Center, One Medical Center Dr, Lebanon, NH 03114 (E-mail: alexander.iribarne@hitchcock.org).

J Thorac Cardiovasc Surg 2023;165:660-1

$0022-5223 / \$ 36.00$

Copyright (c) 2021 by The American Association for Thoracic Surgery

https://doi.org/10.1016/j.jtcvs.2021.04.029
}

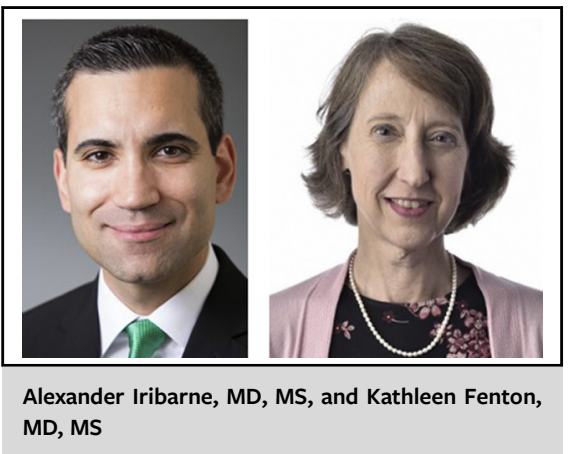

CENTRAL MESSAGE

Significant barriers to broader use of concomitant MAZE exist, but addressing these barriers through continued education, using an implementation science framework, provide opportunity for improvement.

among physicians, patients, health care systems, and populations (translation to real world settings; T4). On average, it takes approximately 17 years for only $14 \%$ of new scientific evidence to enter clinical practice. ${ }^{1}$ Moreover, Americans receive only one half the recommended acute, preventative, and long-term quality health care measures. ${ }^{2}$ As such, the National Heart, Lung, and Blood Institute has identified implementation science, the study of methods to promote the uptake of research findings into routine clinical practice, as an area of high priority for outcomes research. ${ }^{3}$ MehafMehaffey and colleagues ${ }^{4}$ highlight this gap between research and clinical practice through their analysis of 